\title{
Cost analysis of patients undergoing cardiac surgery managed with or without cerebral oximetry (INVOS)
}

\author{
D Walsh ${ }^{1 *}, M$ Bennett ${ }^{2}$, S Bennett ${ }^{2}$ \\ From International Conference for Healthcare and Medical Students 2011 \\ Dublin, Ireland. 4-5 November 2011
}

\section{Introduction}

INVOS measures cerebral oxygen saturation. A 100 patient audit examining the efficacy of INVOS showed a reduction in mortality and post-operative length of stay (LOS) against national statistics [1]. Our aim was to analyse the cost effectiveness of INVOS in mixed and coronary bypass (CABG) only cardiac surgery by comparing the INVOS group to a control group.

\section{Methods}

A cost analysis of the INVOS group was done by comparing against 100 control patients who underwent surgery immediately prior to the INVOS audit. Hospital finance data was used to calculate costs. The areas for comparison were cost of: INVOS equipment; ICU and non-ICU postoperative LOS; stroke rehabilitation. Previous INVOS studies have focused on CABG only patients [2,3]. In order to compare outcomes with those found in these studies we selected the CABG only patients out of the two groups. This data was then analysed to give a final saving for CABG only patients.

\section{Results}

Despite the equipment cost, all other outcomes had a reduced cost in the INVOS group. Overall there was a saving of $£ 102,000$ per 100 patients undergoing cardiac surgery. There was improvement in all outcomes in both INVOS $(n=65)$ and the control group $(n=66)$ once the criteria of CABG only had been applied. LOS was reduced in both groups, and neither group contained an incident of stroke. This resulted in an even greater saving of $£ 114,000$ per 100 CABG only patients. Both comparisons, mixed and CABG only, showed the biggest cost saving to be due to a reduction in post-operative LOS both in ICU and on the wards. This correlates with the findings of previous studies $[2,3]$.

\section{Conclusions}

This cost analysis shows a significant saving when using INVOS in the management of both mixed and CABG only cardiac surgery. The CABG only patients showed the greatest saving of $£ 114,000$ per 100 patients. Previous literature only considered CABG patients and did not undertake any cost analysis. However, further analysis with mixed cardiac surgery and a randomised trial is required.

\section{Author details}

${ }^{1}$ Hull York Medical School, UK. ${ }^{2}$ Cardiothoracic Unit, Castle Hill Hospital, UK.

Published: 9 July 2012

\section{References}

1. Bennett S, Haworth CM, Bennett M, Walsh D: Active management using INVOS data in adult cardiac surgery - An audit 2010. ACTA Brighton; 2010.

2. Slater JP, Guarino $T$, et al: Cerebral oxygen desaturation predicts cognitive decline and longer stay in hospital after cardiac surgery. Ann Thorac Surg 2009, 87:36-45.

3. Murkin JM, et al: Monitoring brain oxygen saturation during coronary bypass surgery: A randomized, prospective study. Anesth Analg 2007, 104:51-58.

doi:10.1186/1753-6561-6-S4-016

Cite this article as: Walsh et al:: Cost analysis of patients undergoing cardiac surgery managed with or without cerebral oximetry (INVOS). BMC Proceedings 2012 6(Suppl 4):016.

${ }^{1}$ Hull York Medical School, UK

Full list of author information is available at the end of the article

(c) 2012 Walsh et al; licensee BioMed Central Ltd. This is an Open Access article distributed under the terms of the Creative Commons Attribution License (http://creativecommons.org/licenses/by/2.0), which permits unrestricted use, distribution, and reproduction in any medium, provided the original work is properly cited. 